\title{
Comparison of Current and Voltage Control Techniques for Neuromuscular Electrical Stimulation in the Anterior Thigh
}

\author{
José L. Vargas Luna ${ }^{1,2}$, Matthias Krenn ${ }^{1}$, Jorge A. Cortés ${ }^{2}$, Winfried Mayr ${ }^{1}$ \\ ${ }^{1}$ Center for Medical Physics and Biomedical Engineering, Medical University of Vienna, Austria \\ ${ }^{2}$ Centro de Innovación en Diseño y Tecnología, Tecnológico de Monterrey, Campus Monterrey, Mexico \\ jl.vargas.phd.mty@itesm.mx
}

\begin{abstract}
A comparison between current control (CC) and voltage control (VC) techniques for functional electrical stimulation (FES) with different electrodes was done. A dependency on current of the ohmic resistance of the skinelectrode interface was found. A controllability study shows that the CC offers more controllability since both amplitude and pulse width $(P W)$ of stimulation are suitable parameters for control, unlike $V C$, where only the amplitude is able to

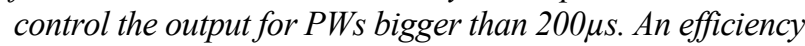
study shows that large PWs are electrically inefficient since not all the applied energy is use for neuromuscular or muscular excitation and up to 50\% of the energy is absorbed by the tissue. Based on these, basic suggestions for parameter selection are given to minimize the risk of tissue damage and improve electric efficiency at least for our specific set-up.
\end{abstract}

Keywords: Current-control, voltage-control, skinelectrode interface impedance, efficiency

\section{Introduction}

Neuromuscular electrical stimulation is well established for rehabilitation and diagnostics. The stimulator design is in most cases based on voltage controlled (VC) or current controlled (CC) output stages. VC devices are considered to be safer than $\mathrm{CC}$ for transcutaneous applications, because loss electrode contact surface does not lead to dangerous high current density. On the other hand, motor unit recruitment under CC stimulation is more reliable, most likely due to the fact that the delivered current is more or less independent from electrode impedance.

However, many studies demonstrate that the skinelectrode interface impedance plays an important role in the stimulation dynamics, and several models have been proposed as equivalent circuits [1], [2]. It is aimed that this impedance variations indirectly affect both kinds of stimulation in effectiveness and controllability.

An important aim of this work was to identify the differences in the control dynamics of both stimulation control techniques and the effects of electrode selection.

\section{Methods}

Multivariable measurements were done in order to investigate the dynamics of the stimulation. $\mathrm{CC}$ and $\mathrm{VC}$ stimulation were applied to 6 subjects using two different surface electrode types of $8 \times 13 \mathrm{~cm}$ (self-adhesive STIMEX, Schwa-Medico GmbH and custom-made conductive rubber electrode). For the $\mathrm{CC}$ stimulation a Stimulette DEN2X (Schuhfried Medizintechnik GmbH, Austria) and for VC stimulation a custom-made device were used. The neuromuscular stimulation was applied transcutaneously on the anterior thigh. The output force was measured with a custom-build dynamometer chair detecting isometric force in three dimensions. Each stimulus was monitored in both voltage and current. Finally, evoked myoelectric signals (M-wave) were recorded from rectus femoris and hamstrings. The EMG electrodes were placed in a transversal disposition instead longitudinally to reduce excessive stimulus artefacts and amplifier overdrive in the EMG recordings.

The whole protocol was divided in four stages: (1) CC with self-adhesive hydrogel electrodes (ETD), (2) VC with ETD, (3) CC with conductive rubber electrodes (ETR) and, (4) VC with ETR.

Stimulation sweeps with pulse widths (PW) modulation from $2 \times 50 \mu$ s to $2 \times 1000 \mu$ s were done. The stimulations amplitudes were varied from the sensory threshold until $\pm 120 \mathrm{~mA}$ (in steps of $5 \mathrm{~mA}$ ) and $\pm 60 \mathrm{~V}$ (in steps of $5 \mathrm{~V}$ ) for $\mathrm{CC}$ and VC respectively. All stimulation impulses were rectangular charge-balanced biphasic pulses.

In order to compare both stimulation techniques the electrical energy applied and the current injection during the whole pulse were considered as parameters. The detected forces were transformed to a $3 \mathrm{D}$ vector and only the magnitude was considered for analysis. The selected equivalent electric circuit for the skin-electrode interface is shown in Fig. 1. The impedance was estimated fitting the curves to the equations that govern this circuit. For the estimation, $\mathrm{R}_{\mathrm{s}}, \mathrm{R}_{\mathrm{p}}$ and $\mathrm{C}$ were considered constant within each pulse, but not between pulses.

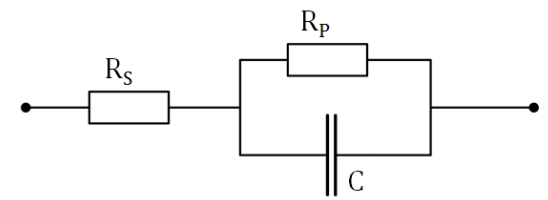

Figure 1: Equivalent model proposed for the skinelectrode interface.

\section{Results}

The impedance approximation was only valid for $\mathrm{CC}$ stimulation, since VC didn't follow the exponential behaviour of the circuit. The impedance results are shown in Fig. 2, only for CC. The recruitment curve is shown in Fig. 3, for both electrodes and techniques. A comparison between charge and energy is shown in Fig. 4, for isotonic curves $(10 \mathrm{~N}$ and $20 \mathrm{~N})$. 


\section{Discussion}

The high dependence (up to 5 times the saturation value) of $R_{p}$ to the stimulation intensity was congruent with other studies [2-4]. $R_{s}$ and $C$ variation due the stimulation amplitude were neglectable. Such dependency was addressed to the current amplitude since only CC stimulation was well-described by the proposed circuit, and system response at a given amplitude and different PWs shows no significant difference.
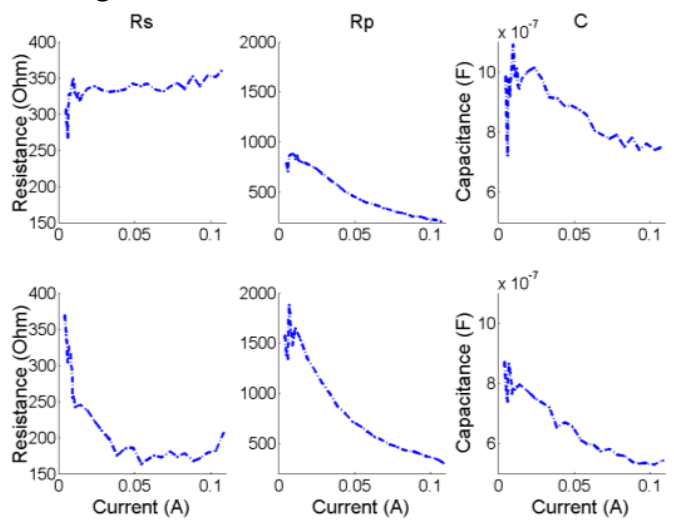

Figure 2: Impedance parameter estimation for CC stimulation with the ETR (upper part) and ETD (lower part) electrodes
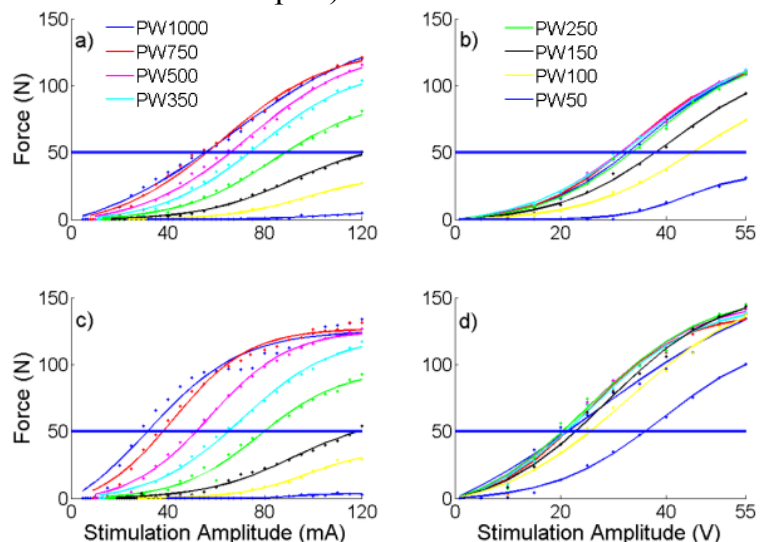

Figure 3: Recruitment curves for a) CC with ETR, b) VC with ETR, c) CC with ETD, and d) VC with ETD

Important differences on $\mathrm{CC}$ and $\mathrm{VC}$ techniques were found by means of its controllability. Both techniques were able to reach similar output force ranges. However, $\mathrm{CC}$ showed better characteristics since both stimulation amplitude and PW are suitable control parameters, unlike VC, were only the amplitude affect the output force significantly on PWs bigger than $200 \mu \mathrm{s}$. This is visible in Fig. 3 for an induced contraction force of $50 \mathrm{~N}$, where it is evident that $\mathrm{CC}$ provides more freedom in control by either PW or amplitude variation than VC, where PW related traces bigger than $200 \mu$ s for ETR and $100 \mu$ s for ETD overlap, and only the variation of the amplitude can lead to the desired output force.

The electric efficiency in both techniques had the same behaviour as seen in Fig. 4. Beyond differences in the magnitude between techniques, it can be observed that, in general, huge PWs require more energy and inject more charge during the stimulus than smaller PWs. This means that energy was absorbed by mechanisms different to neuromuscular stimulation. This absorption leads to a difference of up to $50 \%$ for CC and $35 \%$ for $\mathrm{VC}$ on energy required for obtain the same output force. On the other hand, smaller PWs showed a tendency to require more electric energy to be delivered. For our set-up PW of $200 \mu$ s showed a better performance by means of charge and electric energy delivered, it means a reduction of risk of tissue damage and an improvement in the power efficiency of the device.

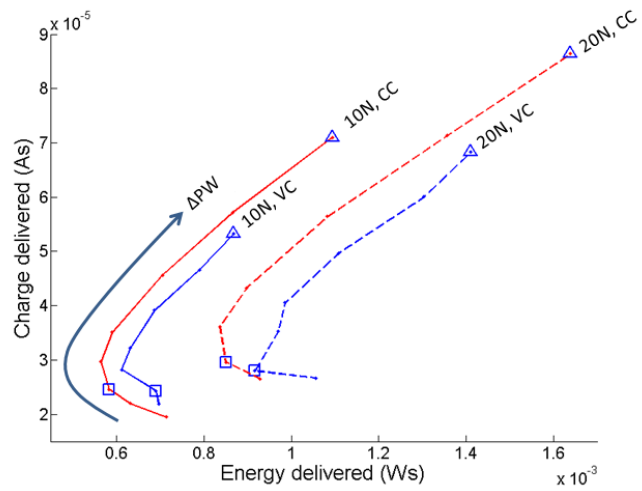

Figure 4: Comparison between necessary charge and energy to generate the stimulation impulse able to induce contraction force of $10 \mathrm{~N}$ (continuous line) and $20 \mathrm{~N}$ (dashed line) with CC (red) and VC (blue). Triangle indicate a duration impulse of $1000 \mu \mathrm{s}$, and the square a duration impulse of $200 \mu \mathrm{s} . \Delta \mathrm{PW}$ arrow indicates direction of $\mathrm{PW}$ increasing

Regardless the current dependency of the impedance, it was observed different variation ranges of it for the two different electrodes. These differences due the material and construction of the electrodes were also reflected in the recruitment curve, showing that both $\mathrm{CC}$ and $\mathrm{VC}$ are dependent on electrode configuration.

Although these results are also valid for our set-up and further research is necessary to generalize these results, the methodology may be useful to optimizing stimulation parameters in various application scenarios.

\section{Bibliography}

[1] G. Kantor, G. Alon, and H. S. Ho, "Simulated tissue loads for testing of transcutaneous electrical stimulators," in Proceedings of 16th Annual International Conference of the IEEE EMBS, pp. 784 785, 1994.

[2] S. J. Dorgan and R. B. Reilly, "A model for human skin impedance during surface functional neuromuscular stimulation.," IEEE transactions on rehabilitation engineering, vol. 7, no. 3, pp. 341-8, Sep. 1999.

[3] D. R. Davis and D. W. Kennard, "Influence of Electric Current on the Skin," Nature, vol. 193, no. 4821, pp. 1186-1187, Mar. 1962.

[4] S. Grimnes, "Skin impedance and electro-osmosis in the human epidermis," Medical \& Biological Engineering \& Computing, vol. 21, no. 6, pp. 739749, Nov. 1983. 